\title{
Approximation of Exterior Dirichlet Problems; Convergence of Wave and Scattering Operators
}

By

Takashi KAKO*

\section{$\S 1$. Introduction}

In the present paper we show that the wave operator in the Dirichlet problem for an elliptic differential operator is the limit of those in the appropriate whole space problems.

The proof of this fact consists of three parts; the convergence of resolvents [Lemmas 1 and 2], the convergence of semi-groups [Lemma 3] and the convergence of wave operators [Theorem 1].

In proving the convergence of resolvents, we generalize the technique used by Ōeda [7]. As for the convergence of semi-groups, we apply a standard method (Kato [2]). And we prove the convergence of wave operators by means of the time-dependent scattering theory. The convergence of scattering operators is also considered [Corollary 1]. We give a comment on the Neumann problem for $-\Delta+q(x)$ [Remark 3].

\section{§2. Statement of Results}

Throughout the present paper we use the following notations: $D_{j}=-i \frac{\partial}{\partial x_{j}}, x=\left(x_{1}, \ldots, x_{n}\right) \in R^{n}$, a multi-index $\alpha=\left(\alpha_{1}, \ldots, \alpha_{n}\right),|\alpha|=\alpha_{1}+$ $\ldots+\alpha_{n}$ and $D^{\alpha}=D_{1}^{\alpha_{1}} \ldots D_{n}^{\alpha_{n}}$. We denote by $H^{m}(\Omega)$ the Sobolev space of order $m$ for a domain $\Omega$ and by $\stackrel{\circ}{H}^{m}(\Omega)$ the closed subspace of $H^{m}(\Omega)$ which is the completion of $C_{\mathrm{o}}^{\infty}(\Omega)$ by the Sobolev norm of order $m$, where $C_{\mathrm{o}}^{\infty}(\Omega)$ is the set of all infinitely differentiable functions with compact support in $\Omega$.

Communicated by S. Matsuura, July 14, 1973.

* Department of Pure and Applied Sciences, College of General Education, University of Tokyo, Tokyo. 
We denote the inner product in $L^{2}\left(R^{n}\right)$ by $($,$) . We denote the domain$ of a form $h[f, g]$ by $D[h]$ and the domain and the range of an operator $A$ by $D(A)$ and $R(A)$ respectively. And we denote the adjoint of an operator $A$ by $A^{*}$. We now consider the self-adjoint operators $H$ and $H^{\circ}$ in $L^{2}\left(R^{n}\right)$ associated with closed elliptic Hermitian symmetric forms $h[f, g]$ and $h^{\circ}[f, g]$ respectively. The exact definitions and expressions of these operators and forms are given as follows. Let $h[f, g]$ and $h^{\circ}[f, g]$ be the sesqui-linear forms defined as

$$
\begin{aligned}
& D[h]=D\left[h^{\circ}\right]=H^{m}\left(R^{n}\right), \\
& h[f, g]=h^{\circ}[f, g]+\left(\sum_{|\alpha|,|\beta| \leq m} a_{\alpha \beta}(x) D^{\beta} f, D^{\alpha} g\right), \quad f, g \in D[h],
\end{aligned}
$$

and

$$
h^{\circ}[f, g]=\left(\sum_{|\alpha|,|\beta| \leqq m} a_{\alpha \beta}^{\circ} D^{\beta} f, D^{\alpha} g\right), \quad f, g \in D\left[h^{\circ}\right] .
$$

On the coefficients $a_{\alpha \beta}^{\circ}$ and $a_{\alpha \beta}(x)$ we impose the following conditions:

$$
\begin{aligned}
& a_{\alpha \beta}^{\circ} \text { are constants, } \\
& \sum_{|\alpha,| \beta \mid=m} a_{\alpha \beta}^{\circ} \xi^{\alpha+\beta} \geqq c|\xi|^{2 m}, \quad a_{\alpha \beta}^{\circ}=\overline{a_{\beta \alpha}^{\circ}}, \\
& \sum_{|\alpha,,| \beta \mid=m}\left\{a_{\alpha \beta}^{\circ}+a_{\alpha \beta}(x)\right\} \xi^{\alpha+\beta} \geqq c|\xi|^{2 m}, \quad a_{\alpha \beta}(x)=\overline{a_{\beta \alpha}(x)}, \\
& a_{\alpha \beta}(x) \text { are bounded measurable functions and } \\
& a_{\alpha \beta}(x) \text { with }|\alpha|=|\beta|=m \text { are uniformly continuous. }
\end{aligned}
$$

Then, by virtue of the Gårding inequality, $h[f, g]$ and $h^{\circ}[f, g]$ are closed elliptic Hermitian symmetric forms bounded from below. We define the self-adjoint operators $H$ and $H^{\circ}$ by these forms $h[f, g]$ and $h^{\circ}[f, g]$ respectively in the sense of Friedrichs. The operators $H$ and $H^{\circ}$ are lower semi-bounded and in particular, for some real constant $\gamma$,

$$
(H f, f) \geqq(-\gamma+1)(f, f), \quad f \in D(H) .
$$

We define the self-adjoint operator $H_{d}$ in $L^{2}(\Omega)$ by the form $h_{d}[f, g]=$ $h[f, g]$ with domain $\stackrel{\circ}{H}^{m}(\Omega)$ in the sense of Friedrichs. Here and hereafter we assume that $\Omega$ is the exterior of a compact set $K$ with smooth boundary $\partial \Omega$. We define the sequence of self-adjoint operators $\left\{H_{n}\right\}$, which approximates $H_{\boldsymbol{d}}$, as follows: 


$$
\begin{aligned}
& D\left(H_{n}\right)=D(H), \\
& H_{n} f=H f+n \chi_{K} f, \quad f \in D\left(H_{n}\right),
\end{aligned}
$$

where $\chi_{K}$ is the characteristic function of $K$.

Next, to state the main theorem, we need the following condition.

Condition $A$. There exist a set $F \subset D\left(H^{\circ}\right)$ which is dense in $L^{2}\left(R^{n}\right)$, an infinitely differentiable function $\eta(x)$ and a constant $R^{\circ}$ such that: i) $\eta(x)=1$ on $\left\{x \|_{1} x \mid>R^{\circ}+1\right\}, \eta(x)=0$ on $\left\{x \| x \mid<R^{\circ}\right\}$ and $\left\{x \|_{1} x \mid<R^{\circ}\right\} \supset K$, and ii) for any $u \in F, \eta(x) e^{-i \tau H^{\circ}} u \in D\left(H^{\circ}\right) \Gamma D(H)$ and the integral

$$
\int_{-\infty}^{\infty}\left\|\left(H \eta(x)-\eta(x) H^{\circ}\right) e^{-i \tau H^{\circ}} u\right\| d \tau
$$

is finite.

Let $J$ be the bounded operator from $L^{2}\left(R^{n}\right)$ to $L^{2}(\Omega)$ given by restriction of a function. Then we obtain the following theorem.

Theorem 1. Under Condition $A$, the wave operators $W_{ \pm}\left(H_{n}, H^{\circ}\right)$ and $W_{ \pm}\left(H_{d}, H^{\circ}\right)$ given by

$$
W_{ \pm}\left(H_{n}, H^{\circ}\right)=\mathrm{s}-\lim _{t \rightarrow \pm \infty} e^{i t H_{n}} e^{-i t H^{\circ}}
$$

and

$$
W_{ \pm}\left(H_{d}, H^{\circ}\right)=\mathrm{s}-\lim _{t \rightarrow \pm \infty} e^{i t H_{d}} \int e^{-i t H^{\circ}}
$$

exist. And

$$
W_{ \pm}\left(H_{d}, H^{\circ}\right)=\mathrm{s}-\lim _{n \rightarrow \infty} \int W_{ \pm}\left(H_{n}, H^{\circ}\right)
$$

Corollary 1. If $W_{ \pm}\left(H_{n}, H^{\circ}\right)$ and $W_{ \pm}\left(H_{d}, H^{\circ}\right)$ are complete, that is,

$$
R\left(W_{+}\left(H_{n}, H^{\circ}\right)\right)=R\left(W_{-}\left(H_{n}, H^{\circ}\right)\right)
$$

and

$$
R\left(W_{+}\left(H_{d}, H^{\circ}\right)\right)=R\left(W_{-}\left(H_{d}, H^{\circ}\right)\right),
$$

then the scattering operator $S_{d}=W_{+}\left(H_{d}, H^{\circ}\right)^{*} W_{-}\left(H_{d}, H^{\circ}\right)$ for the Dirichlet problem is the strong limit of a sequence of operators $S_{n}=W_{+}\left(H_{n}\right.$, $\left.H^{\circ}\right)^{*} W_{-}\left(H_{n}, H^{\circ}\right)$ when $n$ tends to infinity. 
Remark 1. Condition A is satisfied if, for example, the Hessian of $P_{2 m}(\xi)=\sum_{|\alpha|=|\beta|=m} a_{\alpha \beta}^{\circ} \xi^{\alpha+\beta}$ is not identically zero, $a_{\alpha \beta}(x)$ are differentiable to order $|\alpha|$ for $|x|>R>R^{\circ}$ and satisfy $\sum_{|\gamma| \leqq|\alpha|}\left|D^{r} a_{\alpha \beta}(x)\right| \leqq \frac{c}{(1+|x|)^{1+\bar{\varepsilon}}}, \varepsilon>0$, there. The proof of this fact is shown by the stationary phase method (Murata [6]).

Remark 2. The assumed completeness of wave operators in Corollary 1 is proved in Kuroda [3], [4] and [5] under the same condition as in Remark 1.

To prove the theorem, we need the following lemmas.

Lemma 1. Let $R_{n}(\lambda)$ be the resolvent of $H_{n}$, then the strong limit

$$
\mathrm{G}(\lambda)=\mathrm{s}-\lim _{n \rightarrow \infty} R_{n}(\lambda)
$$

exists.

Lemma 2. If we denote by $R_{d}(\lambda)$ the resolvent of $H_{d}$, we have

$$
R_{d}(\lambda)=J G(\lambda) J^{*}=\mathrm{s}-\lim _{n \rightarrow \infty} J R_{n}(\lambda) J^{*}
$$

and

$$
G(\lambda)=J^{*} R_{d}(\lambda) J, \quad G(\lambda) J^{*}=J^{*} R_{d}(\lambda)
$$

Lemma 3. Let $H_{d}$ and $H_{n}$ be as above, then for any fixed twe have

$$
c^{i t H_{d}}=\mathrm{s}-\lim _{n \rightarrow \infty} J e^{i t H_{n}} J^{*} .
$$

Remark 3. The Neumann problem for $-\Delta+q(x)$ : To approximate the Neumann problem for $-\Delta+q(x)$, we can use the following sequence of operators $\left\{H_{n}\right\}$. Let $h_{n}[f, g]$ be the closed Hermitian symmetric forms given by

$$
h_{n}[f, g]=\int_{R^{n}}\left(1-\rho_{n}(x)\right) \nabla f \cdot \overline{\nabla g} d x+\int_{\Omega} q(x) \bar{g} d x
$$

with domain $D\left[h_{n}\right]=H^{1}\left(R^{n}\right)$, where $\rho_{n}(x)$ are all in $C_{\circ}^{\infty}\left(K^{i n t}\right)$ and monotone-increasing in $n$ to 1 in $K^{i n t}$, the interior of $K$. The sequence of 
approximating operators $\left\{H_{n}\right\}$ is defined by these forms $\left\{h_{n}\right\}$ in the sense of Friedrichs. Then all the preceding results also hold in this situation. In fact, the sequence of forms $h_{n}[u, u]$ is monotone-decreasing and converges to a form $\stackrel{\circ}{h}_{N}[u, u]$, where $\stackrel{\circ}{h}_{N}[f, g]$ is the closed Hermitian symmetric form given by

$$
\ddot{h}_{N}[f, g]=\int_{\Omega} \nabla f \cdot \overline{\nabla g} d x+\int_{\Omega}^{0} q\left(x^{*}\right) f \bar{g} d x
$$

with domain $D\left[\stackrel{\circ}{h}_{N}\right]=H^{1}(\Omega) \oplus L^{2}(K)$, the direct sum of $H^{1}(\Omega)$ and $L^{2}(K)$. As this convergence is valid for the element belonging to $H^{1}\left(R^{n}\right)$ which is a core of $\stackrel{\circ}{h}_{N}[f, g]$, the sequence $\left\{H_{n}\right\}$ converges to $\left(H_{N} \oplus 0\right)$, the direct sum of operators $H_{N}$ in $L^{2}(\Omega)$ and 0 in $L^{2}(K)$, in the generalized sense (see Kato [2] VIII $\S 3.2$ ). Here $H_{N}$ is $-\Delta+q(x)$ in $\Omega$ with the Neumann boundary condition. That is, the sequence of resolvents $R_{n}(-\gamma)=$ $\left(H_{n}+\gamma\right)^{-1}$ converges to $\left(\left(H_{N} \oplus 0\right)+\gamma\right)^{-1}=\left(H_{N}+\gamma\right)^{-1} \oplus \gamma^{-1}$. From this fact, the same result as in Lemma 1 follows immediately and the same results as in Lemmas 2, 3 and Theorem 1 are also valid.

\section{§. Proof of Results}

i) Proof of Lemma 1: From the construction of an approximating series $\left\{H_{n}\right\}$,

$$
\left(H_{m}+\gamma\right) \geqq\left(H_{n}+\gamma\right) \geqq(H+\gamma) \geqq 1, \quad m \geqq n,
$$

so that

$$
1 \geqq R_{n}(-\gamma) \geqq R_{m}(-\gamma) \geqq 0
$$

Accordingly, we obtain Lemma 1 by a well-known result (Kato [2] VIII $\S 3.1)$.

ii) Proof of Lemma 2: Put $\left(H_{n}+\gamma\right)^{-1} u=f_{n}$. Then

$$
\begin{aligned}
1 & \left(R_{n}(-\gamma) u, u\right) \\
& =\left(\left(H_{n}+\gamma\right) f_{n}, f_{n}\right) \geqq h\left[f_{n}, f_{n}\right]+\gamma\left\|f_{n}\right\|^{2} \geqq c\left(h^{\circ}\left[f_{n}, f_{n}\right]+\gamma\left\|f_{n}\right\|^{2}\right) .
\end{aligned}
$$

This shows $\left\{f_{n}\right\}$ is a bounded set in $H^{m}\left(R^{n}\right)=D\left[h^{\circ}\right]$. On the other hand, as $\left\{f_{n}\right\}$ converges to $G(-\gamma) u=g$ in $L^{2}\left(R^{n}\right)$ by Lemma $1, g \in H^{m}\left(R^{n}\right)$ and 
$\left\{f_{n}\right\}$ converges weakly to $g$ in $H^{m}\left(R^{n}\right)$ (see, for example, Agmon [1] $\S 3$. Theorem 3.12). Furthermore, as

$$
1 \geqq\left(\left(H_{n}+\gamma\right) f_{n}, f_{n}\right) \geqq n\left(\chi_{K} f_{n}, f_{n}\right),
$$

$\left\{\chi_{K} f_{n}\right\}$ converges to zero and hence $\left.g\right|_{K}=0$. Then $J g$, the restriction of $g \in H^{m}\left(R^{n}\right)$ to $\Omega$, is in $\stackrel{\circ}{H}^{m}(\Omega)$. Consequently, $J G(-\gamma) J^{*} v \in \stackrel{\circ}{H}^{m}(\Omega)$ for $v \in L^{2}(\Omega)$. Now let $v \in L^{2}(\Omega)$ and $\phi \in \stackrel{\circ}{H}^{m}(\Omega)$, then

$$
\begin{aligned}
& \left(h_{d}+\gamma\right)\left[J G(-\gamma) J^{*} v, \phi\right]=(h+\gamma)\left[G(-\gamma) J^{*} v, J^{*} \phi\right] \\
& \quad=\lim _{n \rightarrow \infty}(h+\gamma)\left[\left(H_{n}+\gamma\right)^{-1} J^{*} v, J^{*} \phi\right] \\
& \quad=\left(J^{*} v, J^{*} \phi\right)-\lim _{n \rightarrow \infty} n\left(\chi_{K}\left(H_{n}+\gamma\right)^{-1} J^{*} v, J^{*} \phi\right)=\left(J J^{*} v, \phi\right)=(v, \phi) .
\end{aligned}
$$

In particular, for $\psi \in D\left(H_{d}\right) \subset \stackrel{\circ}{H}^{m}(\Omega)$ we have

$$
\left(J G(-\gamma) J^{*} v,\left(H_{d}+\gamma\right) \psi\right)=(v, \psi)=\left(\left(H_{d}+\gamma\right)^{-1} v,\left(H_{d}+\gamma\right) \psi\right) .
$$

Then, as $\left\{\left(H_{d}+\gamma\right) \psi \mid \psi \in D\left(H_{d}\right)\right\}=L^{2}(\Omega)$, we have

$$
J G(-\gamma) J^{*}=\left(H_{d}+\gamma\right)^{-1}=R_{d}(-\gamma) .
$$

This proves the first part of Lemma 2. To prove the second part, we multiply this equation by $J$ and $J^{*}$ from right and left:

$$
J^{*}\left(H_{d}+\gamma\right)^{-1} J=J^{*} J G(-\gamma) J^{*} J
$$

On the other hand

$$
G(-\gamma)-J^{*} J G(-\gamma) J^{*} J=G(-\gamma)\left(1-J^{*} J\right)+\left(1-J^{*} J\right) G(-\gamma) J^{*} J
$$

The second term of the righthand side of this equation is zero since $R(G(-\gamma)) \subset\left\{g \in L^{2}\left(R^{n}\right)|g|_{K}=0\right\}$. The first term is also zero, because for $u$ with support in $K$ and $f_{n}=\left(H_{n}+\gamma\right)^{-1} u$,

$$
u=\left(H_{n}+\gamma\right) f_{n}=\chi_{K}\left(H_{n}+\gamma\right) f_{n}
$$

so that

$$
\left\|f_{n}\right\|^{2} \leqq\left(\left(H_{n}+\gamma\right) f_{n}, f_{n}\right)=\left(u, \chi_{K} f_{n}\right) \leqq\|u\| \cdot\left\|\chi_{K} f_{n}\right\|
$$

and, as $\lim _{n \rightarrow \infty}\left\|\chi_{K} f_{n}\right\|^{\frac{1}{2}}=0$, 


$$
\|G(-\gamma) u\|=\lim _{n \rightarrow \infty}\left\|f_{n}\right\| \leqq\|u\|^{\frac{1}{2}} \cdot \lim _{n \rightarrow \infty}\left\|\chi_{K} f_{n}\right\|^{\frac{1}{2}}=0
$$

Hence

$$
J^{*}\left(H_{d}+\gamma\right)^{-1} J=J^{*} J G(-\gamma) J^{*} J=G(-\gamma) .
$$

iii) Proof of Lemma 3: Using Lemma 2, we can show this lemma by a standard method of expressing the difference of two semi-groups by an integral formula (see Kato [2] IX § 2.4):

$$
\begin{aligned}
& R_{n}(-\gamma)\left(e^{i t H_{n}} J^{*}-J^{*} e^{i t H_{d}}\right) R_{d}(-\gamma) u
\end{aligned}
$$

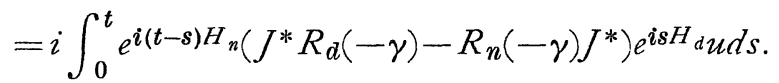

By Lemmas 1 and 2, the righthand side tends to zero as $n \rightarrow \pm \infty$. Therefore, using Lemma 2 and the uniform boundedness of $R_{n}(-\gamma)$ and $e^{i t H_{n}}$, we obtain the desired result.

iv) Proof of Theorem 1: Put

$$
f_{u}(t)=\left\|\left(H \eta(x)-H^{\circ} \eta(x) e^{i t H^{\circ}}\right) u\right\|^{\prime}, \text { for } \quad u \in F .
$$

Then, $f_{u}(t)$ is integrable and

$$
\begin{aligned}
& \left\|\left(e^{i t H_{n}} \eta(x) e^{-i t H^{\circ}}-e^{i s H_{n}} \eta(x) e^{-i s H^{\circ}}\right) u\right\| \\
& \quad=\| \int_{s}^{t} e^{i \tau H_{n}\left(H_{n} \eta(x)-\eta(x) H^{\circ}\right) e^{-i \tau H^{\circ}} u d \tau \|} \\
& \quad \leqq \int_{s}^{t}\left\|\left(H \eta(x)-\eta(x) H^{\circ}\right) e^{-i \tau H^{\circ}} u\right\| d \tau=\int_{s}^{t} f_{u}(\tau) d \tau .
\end{aligned}
$$

The existence of $W_{ \pm}\left(H_{n}, H^{\circ}\right)$ follows immediately from the fact that

$$
\mathrm{s}-\lim _{t \rightarrow \pm \infty} e^{i t H_{n}} e^{-i t H^{\circ}}=\mathrm{s}-\lim _{t \rightarrow \pm \infty} e^{i t H_{n}} \eta(x) e^{-i t H^{\circ}}
$$

which follows from Rellich's compactness theorem. The same argument is valid for $H_{d}$ and $W_{ \pm}\left(H_{d}, H^{\circ}\right)$ exists. Now then, if we let $t$ tend to $\pm \infty$ in $\left(^{*}\right)$, we have

$$
\begin{aligned}
& \left\|\left(J W_{ \pm}\left(H_{n}, H^{\circ}\right)-J e^{i s H_{n}} J^{*} J \eta(x) e^{-i s H^{\circ}}\right) u\right\| \\
& \quad \leqq\left\|\left(W_{ \pm}\left(H_{n}, H^{\circ}\right)-e^{i s H_{n}} \eta(x) e^{-i s H^{\circ}}\right) u\right\| \leqq \int_{s}^{\infty} f_{u}(\tau) d \tau .
\end{aligned}
$$

On the other hand, for $H_{d}$ we have 
$\left(*^{* *}\right) \quad\left\|\left(W_{ \pm}\left(H_{d}, H^{\circ}\right)-e^{i s H_{d}} J \eta(x) e^{-i s H^{\circ}}\right) u\right\| \leqq \int_{s}^{\infty} f_{u}(\tau) d \tau$.

Since the righthand side of $\left(^{* *}\right)$ is independent of $n$, we have, using Lemma 3 and the inequalities $\left({ }^{*}\right)$ and $\left({ }^{* *}\right)$, that for any $\varepsilon>0$, there exists $N$ such that

$$
\begin{aligned}
& \left\|W_{ \pm}\left(H_{d}, H^{\circ}\right) u-J W_{ \pm}\left(H_{n}, H^{\circ}\right) u\right\| \\
& \quad \leqq\left\|\left(W_{ \pm}\left(H_{d}, H^{\circ}\right)-e^{i s H_{d}} J \eta(x) e^{-i s H^{\circ}}\right) u\right\| \\
& \quad+\left\|\left(e^{i s H_{d}}-J e^{i s H_{n}} J^{*}\right) J \eta(x) e^{-i s H^{\circ}} u\right\|^{i} \\
& \quad+\left\|\left(J W_{ \pm}\left(H_{n}, H^{\circ}\right)-J e^{i s H_{n}} \eta(x) e^{-i s H^{\circ}}\right) u\right\|<\varepsilon, \text { for } n>N .
\end{aligned}
$$

Here we choose $s$ sufficiently large so that the first and the third terms are less than $\varepsilon / 3$.

v) Proof of Corollary 1: We have only to show the weak convergence since $S_{n}$ and $S_{d}$ are all unitary. The weak convergence is an immediate consequence of the strong convergence of wave operators.

\section{References}

[1] Agmon, S., Lectures on Elliptic Boundary Value Problems, Princeton, N.J.: D. Van Nostrnd Co., Inc., 1965.

[2] Kato, T., Perturbation Theory for Linear Operators Springer-Verlag, Berlin-HeidelbergNew York, 1966.

[3] Kuroda, S. T., Scattering theory for differential operators, I, operator theory, J. Math. Soc. Japan 25 (1973), 75-104.

[4] Kuroda, S. T., Scattering theory for differential operators, II, self-adjoint elliptic operators, J. Math. Soc. Japan 25 (2) (1973), 222-234.

[5] Kuroda, S. T., Scattering theory for differential operators, III, exterior problems, (to appear).

[6] Murata, M., On the decay of solutions of certain partial differential equations with constant coefficients (unpublished).

[7] Oeda, K., Approximation of obstacles by high potentials; convergence of eigenvalues, Proc. Japan Acad., 46 (1970), 663-667. 